Article

\title{
Investigation on Surface Properties of Mn-Doped CdSe Quantum Dots Studied by X-ray Photoelectron Spectroscopy
}

\author{
Nor Aliya Hamizi ${ }^{1, *}$, Mohd Rafie Johan ${ }^{1}$, Yasmin Abdul Wahab ${ }^{1}{ }^{\circledR}$, Zaira Zaman Chowdhury ${ }^{1}$, \\ Omid Akbarzadeh $\left.{ }^{1}{ }^{(}\right)$, Suresh Sagadevan ${ }^{1}$, Irfan Anjum Badruddin ${ }^{2}{ }^{(D}$, \\ Tatagar Mohammad Yunus Khan ${ }^{2}$ and Sarfaraz Kamangar ${ }^{2}$ \\ 1 Nanotechnology \& Catalysis Research Centre, Deputy Vice Chancellor (Research \& Innovation) Office, \\ University of Malaya, Kuala Lumpur 50603, Malaysia; mrafiej@um.edu.my (M.R.J.); \\ yasminaw@um.edu.my (Y.A.W.); dr.zaira.chowdhury@um.edu.my (Z.Z.C.); omid@um.edu.my (O.A.); \\ sureshsagadevan@gmail.com (S.S.) \\ 2 Department of Mechanical Engineering, College of Engineering, King Khalid University, P.O. Box 394, \\ Abha 61421, Saudi Arabia; magami.irfan@gmail.com (I.A.B.); yunus.tatagar@gmail.com (T.M.Y.K.); \\ sarfaraz.kamangar@gmail.com (S.K.) \\ * Correspondence: aliyahamizi@um.edu.my
}

Received: 21 August 2019; Accepted: 23 September 2019; Published: 7 October 2019

\begin{abstract}
In this work, we report on the effects of incorporating manganese (Mn) dopant into different sizes of cadmium selenide (CdSe) quantum dots (QDs), which improves the electronic and optical properties of the QDs for multiple applications such as light-emitting diodes, lasers, and biological labels. Furthermore, the greener inverse Micelle method was implemented using organic ligand, which is oleic acid. This binding of the surface enhanced the QDs' surface trap passivation of Mn-doped CdSe, which then increased the quantity of the output. In addition, the inverse Micelle technique was used successfully to dope Mn into CdSe QDs without the risk of Mn dopants being self-purified as experienced by wurtzite CdSe QDs. Also, we report the X-ray photoelectron spectroscopy (XPS) results and analysis of zinc blended manganese-doped cadmium selenide quantum dots (Mn-doped CdSe QDs), which were synthesized with physical sizes that varied from 3 to $14 \mathrm{~nm}$ using the inverse Micelle method. The XPS scans traced the existence of the Se $3 \mathrm{~d}$ and Cd $3 \mathrm{~d}$ band of CdSe crystals with a 54.1 and $404.5 \mathrm{eV}$ binding energy. The traced $640.7 \mathrm{eV}$ XPS peak is proof that Mn was integrated into the lattice of CdSe QDs. The binding energy of the QDs was related to the increase in the size of the QDs.
\end{abstract}

Keywords: cadmium selenide; quantum dots; manganese doped; semiconductor; chemical synthesis; and X-ray photoelectron spectroscopy

\section{Introduction}

In semiconductor nanocrystal systems such as CdSe QDs, the transition behavior of the charge carrier on a crystal surface has been found to be crucial in tailoring the electronic and optical properties of CdSe QDs. However, pure QDs such as CdSe, experience surface defects that act as a charge carrier trap, which results in an increase in the probability of non-radiative recombination that decreases the quantity of the fluorescence output [1]. Moreover, like many other QDs, CdSe QDs have a large surface area, resulting in larger surface energy. Therefore, it is volatile or metastable thermodynamically [2]. Researchers have made considerable effort to address this issue by incorporating dopants into the surface of CdSe QDs [3]. Doping the surface of QDs using a lower transition metal (i.e., $\mathrm{Mn}, \mathrm{Mg}$, and $\mathrm{Zn}$ ) is one of the easiest ways to change the wavelength of light emission [4,5]. However, doping $\mathrm{Mn}^{2+}$ into 
CdSe QDs is limited due to the self-purification of QDs with a wurtzite structure [6]. Mn-doped CdSe QDs (zinc blend) have been very significant in semiconductor studies because the incorporation of $\mathrm{Mn}$ into CdSe QDs promises a high-density diluted semiconductor for spintronic application, and provides excellent traps for electrical excitation, which is important for electronic and optoelectronic devices [7]. Tuning the size of the QDs [8] to target a particular application requirement can obtain the bandgap tunability. Integrating size variability and introducing transition metal dopant into the CdSe QDs system has attracted a good deal of attention, especially with regard to the analysis of results and how it can be manipulated in the particular application. Surface-sensitive quantitative spectroscopic technique such as XPS have been found to be an essential part of this analysis as it provides information on the elemental composition of the parts per thousand range, the empirical formula, and the chemical and electronic state of the elements in a material $[9,10]$. Therefore, in this paper, we studied the X-ray photoelectron spectroscopy (XPS) analysis of zinc blended manganese-doped cadmium selenide quantum dots (Mn-doped CdSe QDs) synthesized using the inverse Micelle method.

\section{Experimental Procedure}

Mn-doped CdSe QDs were synthesized using Mn-Cd and Se precursors. Mn acetate $(0.5 \mathrm{~g})$ and $0.5 \mathrm{~g}$ of $\mathrm{CdO}, 25 \mathrm{~mL}$ of paraffin oil and $15 \mathrm{~mL}$ of oleic acid were placed in a three-neck round bottom flask. The mixture was placed into a glove box under vacuum conditions. The solution was heated to $160^{\circ} \mathrm{C}$ and stirred until the $\mathrm{CdO}$ was totally dissolved, obtaining a light yellowish homogeneous solution. Then, $0.079 \mathrm{~g}$ of Se in $50 \mathrm{~mL}$ of paraffin oil was heated to $220^{\circ} \mathrm{C}$ in a glove box under vacuum conditions with fast stirring in another three-neck round bottom flask. The solution turned light orange and then wine red. During the fast stirring, approximately $5 \mathrm{~mL}$ of $\mathrm{Mn}-\mathrm{Cd}$ solution was quickly injected into the Se solution. Immediately after the injection, the temperature dropped to $210{ }^{\circ} \mathrm{C}$ and then rose to $220^{\circ} \mathrm{C}$. For the growth of CdSe QDs at different times, i.e., 0, 0.2, 0.5, 1, 5, 16, 46 and $90 \mathrm{~min}$, the temperature was maintained at $220^{\circ} \mathrm{C}$. Finally, the precipitate was isolated from the solvents by centrifugation, and the unreacted reagent was washed several times with methanol and then dried in the $50^{\circ} \mathrm{C}$ vacuum oven.

\subsection{Physical Observations}

Figure 1 shows the vials of Mn-doped CdSe QD solution. The color variations during the reaction time were affected by the change in the size of the Mn-doped CdSe QDs. The observed colors are due to the strong photon absorption by the Mn-doped CdSe QDs at a particular frequency. The sample with an instant reaction time $(0 \mathrm{~min})$ has a light orange color, as shown in Figure 1a, while the solution with a reaction times of $0.2 \mathrm{~min}$ has a more intense orange color, as shown in Figure $1 \mathrm{~b}$. At a reaction time of 0.5 and $1 \mathrm{~min}$ (Figure 1c,d), the sample appears red. As the reaction times increased (16-90 min), the color turned to dark brown, as shown in Figure 1f,g, and the solution became light brown as the dark brown precipitates settled on the bottom of the containers. Due to the quantum confinement effect, the color differences are ascribed to the variation in the size of the QDs. Larger QDs emit longer wavelengths and smaller QDs emit shorter wavelengths, resulting in a different color emission [11].

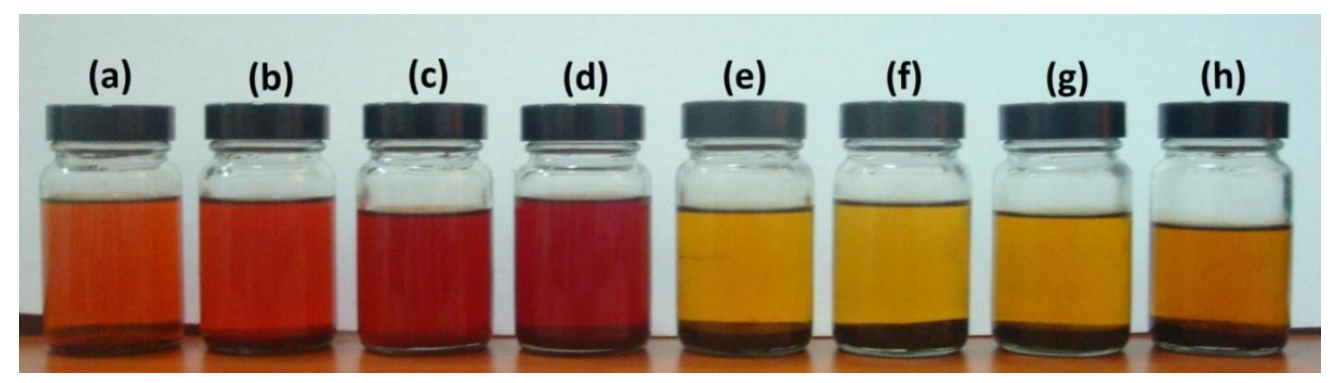

Figure 1. Manganese-doped cadmium selenide quantum dots (Mn-doped CdSe QDs) samples with various colors at different reaction times: (a) 0, (b) 0.2, (c) 0.5, (d) 1, (e) 5, (f) 16, (g) 46 and (h) $90 \mathrm{~min}$. 


\subsection{Characterization Techniques}

Determination of the particle size of Mn-doped CdSe QDs was conducted using a LIBRA 120 PLUS (Carl Zeiss, Oberkochen, Germany) instrument with an energy filtered transmission electron microscope (EFTEM). Structural tests of Mn-doped CdSe QD samples were conducted using an Empyrean X-ray diffractometer (XRD) (Malvern Panalytical Ltd., Malvern, United Kingdom) fitted with graphite monochromatized $\mathrm{Cu} \mathrm{K} \alpha$ radiation $(\lambda=1.54060 \AA$ Á) irradiated with a scanning speed of $0.02^{\circ} \mathrm{s}^{-1}$ and usable range of $2^{\circ}-80^{\circ}$. The elemental compositions and chemical bonding states of solid Mn-doped CdSe QDs samples were observed using Axis Ultra DLD/Kratos/2009 (Kratos Analytical, Manchester, United Kingdom) X-ray Photoelectron Spectrometer (XPS) with 0.1 to 1 atomic \% detection limit and $0.5 \mathrm{~nm}$ depth profiles (under an argon gas environment).

\section{Results and Discussions}

Figure 2 shows typical TEM images for Mn-doped CdSe QDs. The TEM images show that for all reaction times, Mn-doped CdSe QDs have a quasi-sphere shape. Due to the high density of particles, dark areas formed in the image. The mean size and distributions of the QDs are evaluated by one hundred particles as plotted in the histogram (insert) in the respective figure. The average physical size of the Mn-doped CdSe QDs ranged from 3 to $14 \mathrm{~nm}$ for 0 to 90 min reaction time, respectively. This finding demonstrates that there is a slight increase in the physical size of QDs in Mn-doped CdSe QDs compared to pure CdSe QDs [10]. The significantly larger size of Mn-doped CdSe QDs compared to pure CdSe QDs may be as a result of Mn which contributes to shelling the core of the CdSe QDs [3].

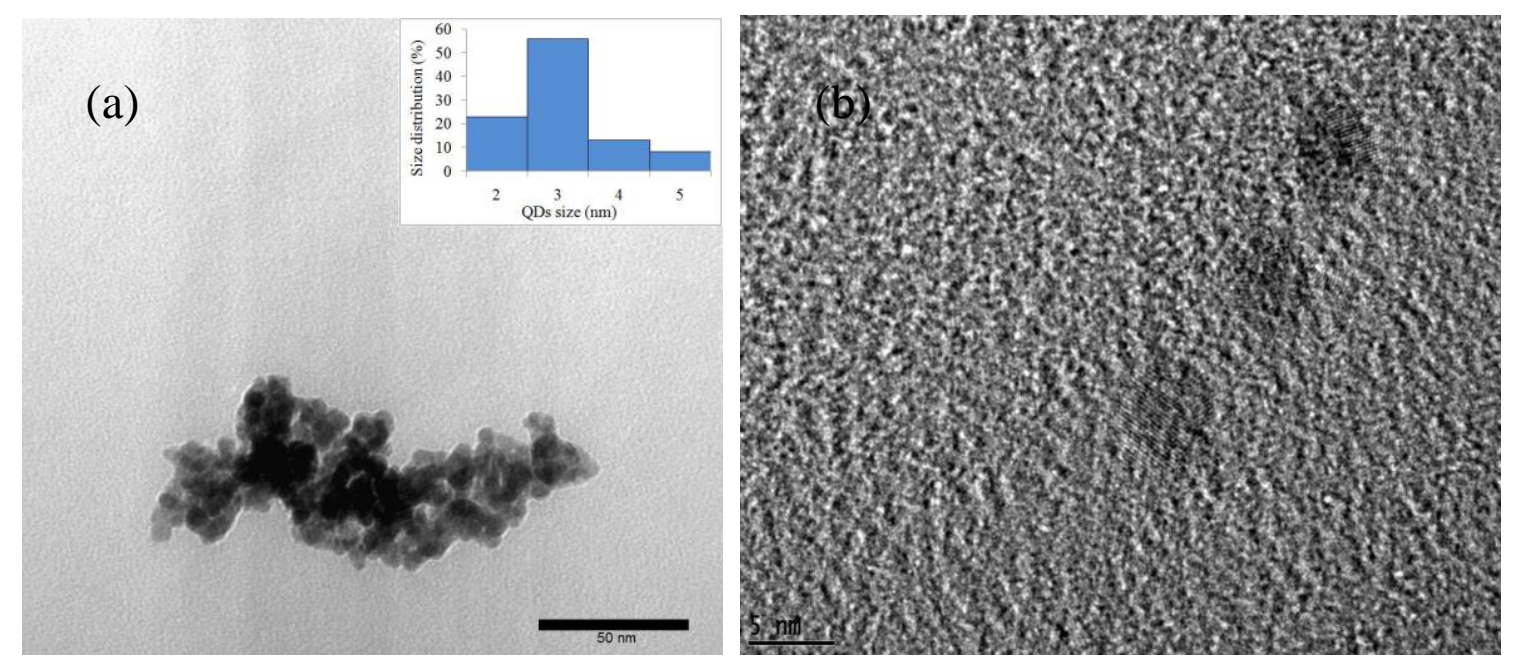

Figure 2. (a) TEM image of Mn-doped CdSe QDs of 0.2 min sample with QDs size distribution (insert) and (b) the lattice structure image of 0.2 min sample.

The relationship between QD growth and reaction time is shown in Figure 3. The growth of QDs at the early reaction time ( 0 to $0.5 \mathrm{~min}$ ) is very important. The fastest growth was during the transition time from 0.2 to $0.5 \mathrm{~min}$, as the high-energy surface was largely used to initiate the initial stage nucleation of Mn-doped CdSe QDs (0-0.2 min). As the reaction time increases, the growth becomes less prominent, particularly from 0.5 to $90 \mathrm{~min}$. This is due to the decrease in the ionic concentration of the chemical precursors in the solution as the time gets longer [4,12]. Furthermore, a narrow particle-size distribution was noted in each sample. Despite the narrow size distribution, there appears to be a slight increase in the overall size distribution over the reaction time. This produces larger QDs, owing to the agglomeration of particles. At the same time, the development of intrinsic QDs continues owing to the chemical precursor residue. This will generate a wide QDs size distribution. The tendency to agglomerate can be compared to the core or bulk of QDs throughout the heating process due to the 
excessively high surface energy on the surface of the QDs, which is favorable for the build-up of the surface or the shell of QDs. This means that QDs have a tendency to grow as long as there is heating. Despite this, as the reaction time increases, the concentration of chemical precursors such as $\mathrm{Cd}, \mathrm{Se}$, and Mn decreases. This retards the development of the QDs surface. Therefore, instead of producing QDs, excessive surface energy enhances the propensity of QDs to agglomerate [4].

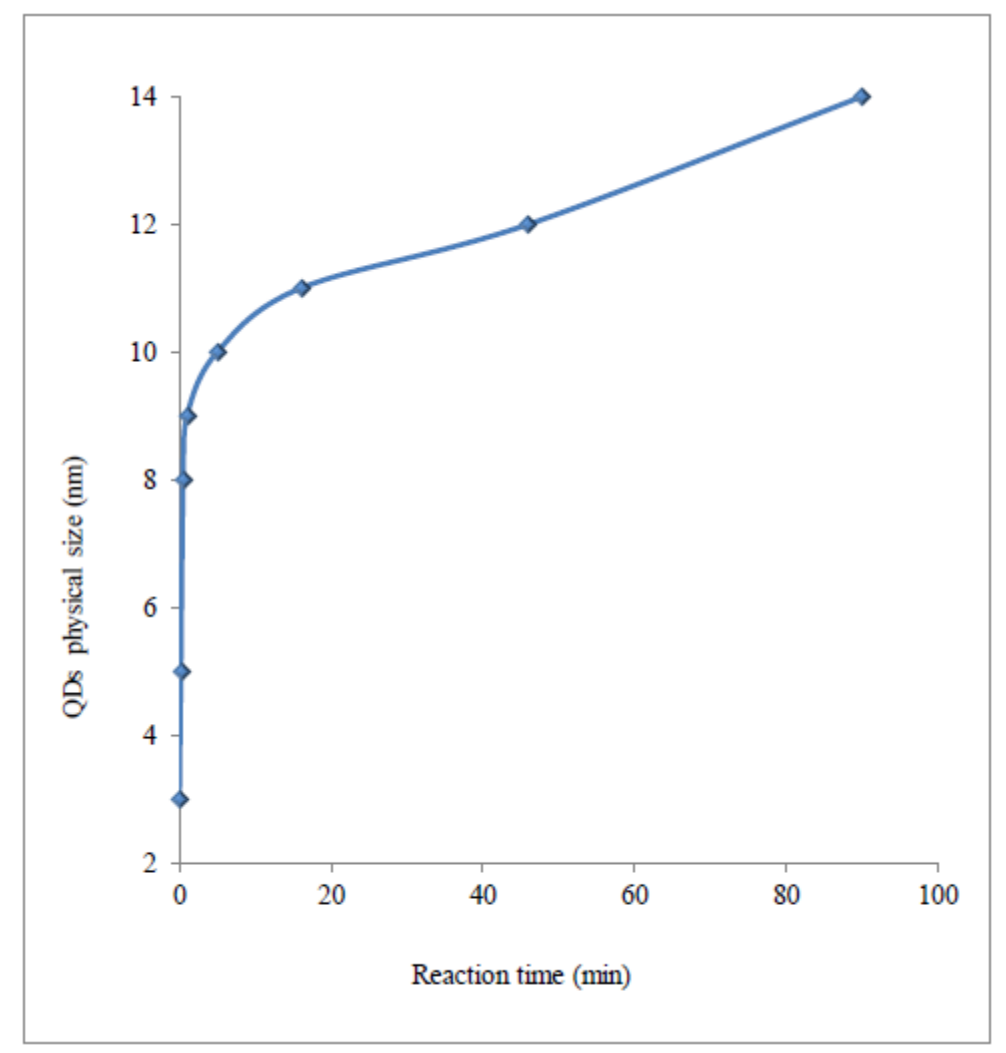

Figure 3. The growth of QDs with reaction times.

As shown in Figure 4, the XRD diffraction pattern of Mn-doped CdSe QDs determined the crystal structure. Three well-defined peaks were noted corresponding to (111), (220) and (311) planes, which is in good agreement with earlier reports [3]. This XRD pattern also demonstrate that as-synthesized Mn-doped CdSe QDs created the zinc blende structure owing to the low temperature of synthesis $\left(220{ }^{\circ} \mathrm{C}\right)$. Due to high synthesis temperatures that go up to $300{ }^{\circ} \mathrm{C}[13]$, other chemical synthesis processes, such as the TOP-based route, produce wurtzite structure.

The surface composition of Mn-doped CdSe QDs was analyzed using X-ray photoelectron spectroscopy (XPS) as shown in Figure 5. The XPS scans traced the presence of the CdSe crystal Se 3d and $\mathrm{Cd} 3 \mathrm{~d}$ band with a binding energy of 54.1 and $404.5 \mathrm{eV}$, respectively [14]. The Mn 2p band from Mn element was found to have a binding energy of roughly $640.7 \mathrm{eV}$ [15], thus confirming the existence of Mn interstitial sites on the surface of CdSe QD's core, acting like a doping element. This background correction to tailor the shift of the peak due to the retarding field at the surface of the specimen is called static charging. The static charging phenomena reduce the kinetic energy of the excited electron [15].

The intensity of the $\mathrm{C} 1 \mathrm{~s}$ and $\mathrm{O} 1 \mathrm{~s}$ peaks were observed to be significant in each scan, with the peaks of both traced elements showing notable intensity in each scan compared to the bands of the other traced elements, including Cd 3d, Se 3d and Mn 2p, which may indicate the influence of oleic acid capping on the surface of the Mn-doped CdSe QDs, which contributed to the formation of Cd-oleate $\left(\mathrm{C}_{36} \mathrm{H}_{70} \mathrm{CdO}_{4}\right)$ and Mn-oleate $\left(\mathrm{C}_{36} \mathrm{H}_{66} \mathrm{MnO}_{4}\right)$ on the surface of QDs, specifically on $\{001\}$ planes. The presence of $\mathrm{Cd}$-oleate and $\mathrm{Mn}$-oleate reduced the dangling orbitals on the surface of QDs, which is the essential point of doping Mn into the CdSe QDs. These dangling orbitals are the orbitals 
that point away from the surface of the QDs and they result from incomplete bonding because of the lower coordination number on the surface, which is contrary to the interior structure of the QDs. In the case of CdSe QDs, both Cd and Se dangling orbitals each act as an electron trap and hole trap, thus, they are able to trap charge carriers on the surface of CdSe QDs, which leads to decreases in the quantum yield [16-18]. Hence, it is assumed that Mn-doped CdSe QDs will have higher fluorescence quantum yield than pure CdSe QDs.

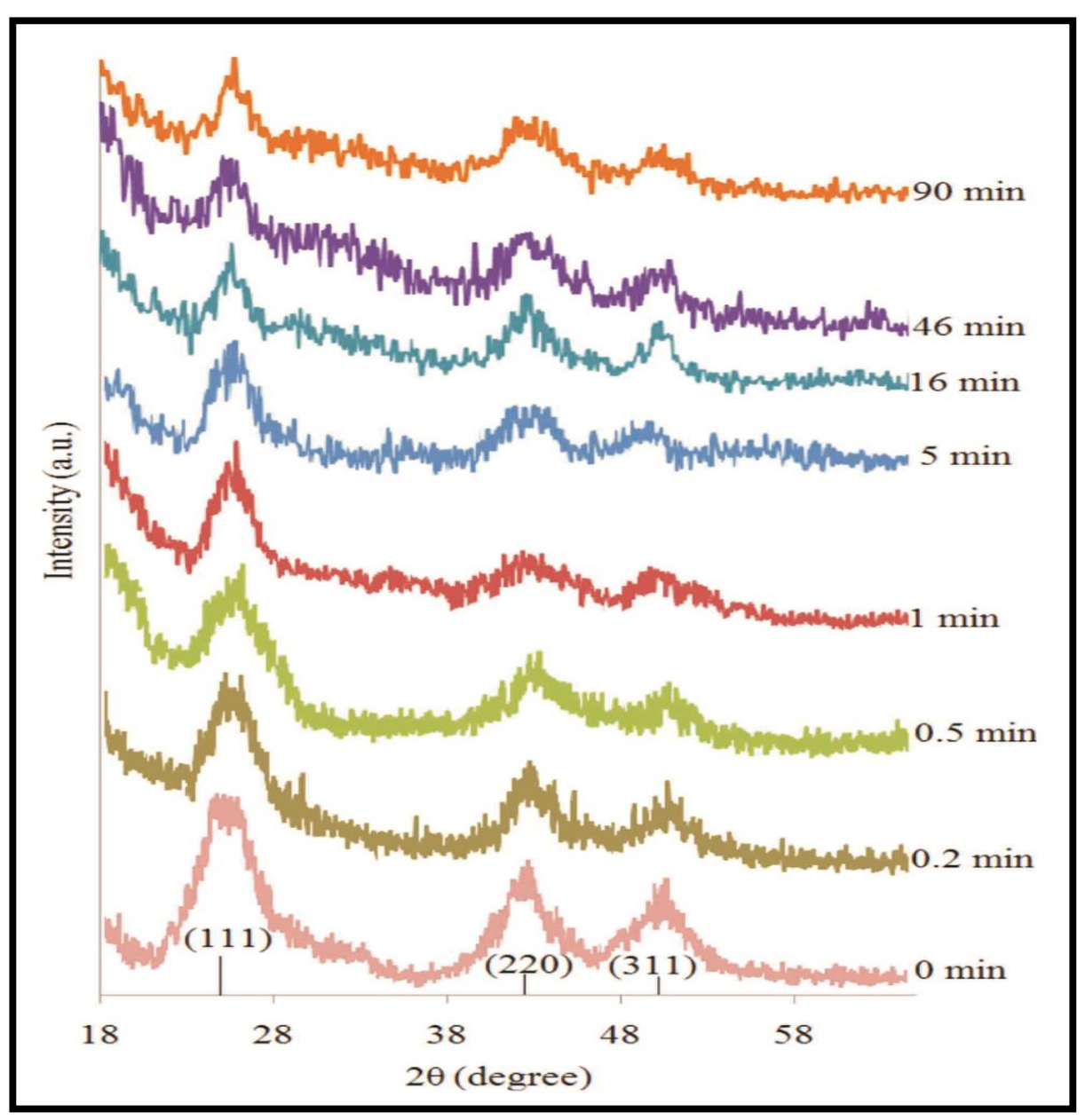

Figure 4. XRD patterns of Mn-doped CdSe QDs sample.

Furthermore, the significant C1s peak may also be associated with the existence of organic residue from the solvent used, which is paraffin that has a general chemical formula of $\mathrm{C}_{x} \mathrm{H}_{(2 x+2)}$. The adsorption of adventitious carbon during the drying and handling process may add a point to the observed C1s peak [16]. Figure 2 provides information about the surface elements, and it also supplies crucial information about the kinetics and interactions between electrons at certain orbitals that contain an unpaired electron. In the initial or original state, the unpaired electron is located at the $3 \mathrm{~d}$ orbital for $\mathrm{Cd}$ and Se, and the 2p orbital for Mn for CdSe Mn-doped QDs. The spin-orbital splitting may occur in the final state when the unpaired electron from ionization during the photoemission process couple with the unpaired electron from the initial or original state at specific spin-orbitals [16]. 


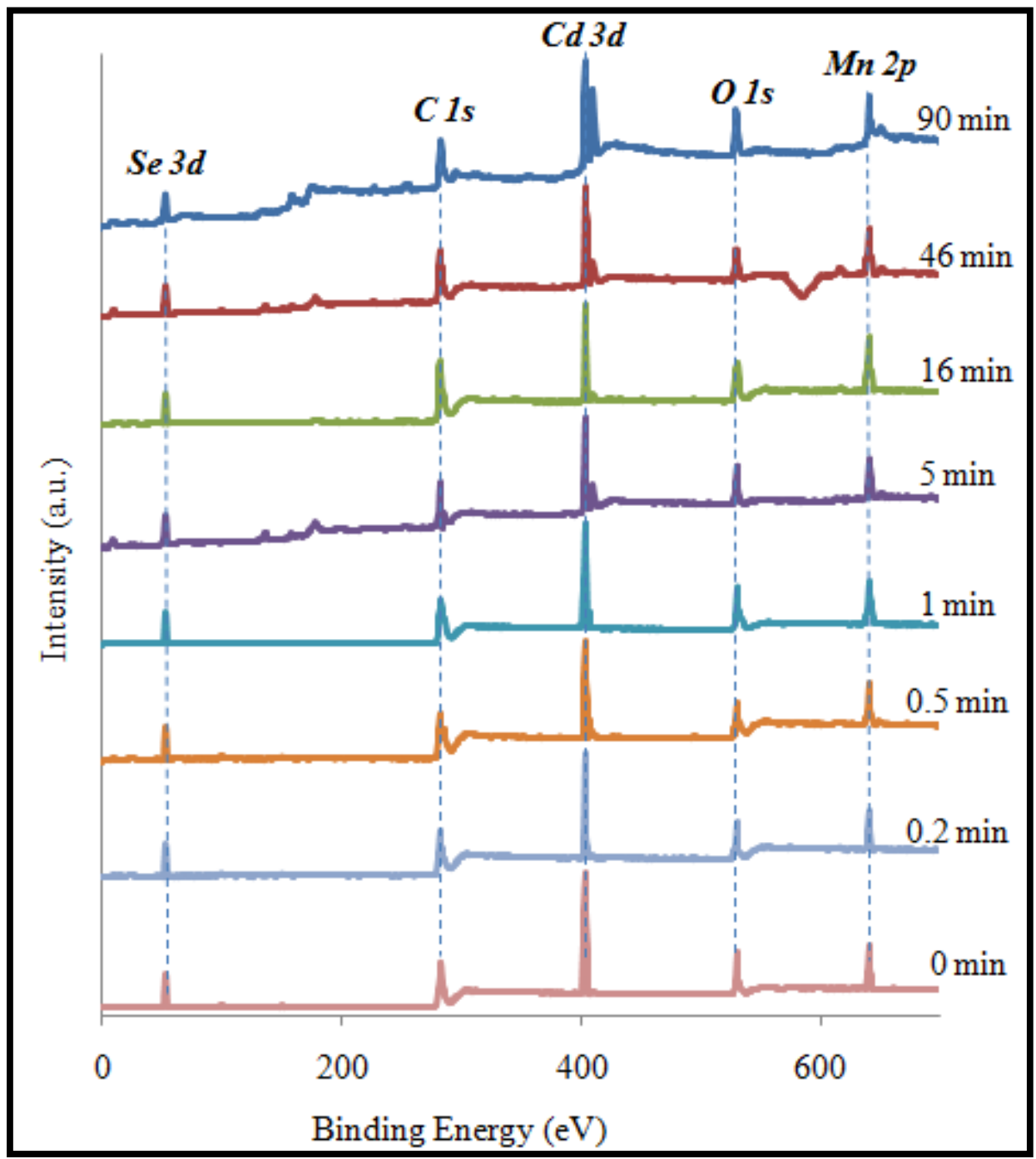

Figure 5. XPS wide scan of Mn-doped CdSe QDs at different reaction times.

Figure 6 shows the $\mathrm{Cd} 3 \mathrm{~d}$ individual peaks of Mn-doped CdSe QDs for different reaction times. The maximum peak intensity (original spectra) shifts towards higher binding energy as the reaction time increases. This suggests that the chemical shift is a function of the size of the Mn-doped CdSe QDs [19]. Figure 6 also shows $\mathrm{Cd} 3 \mathrm{~d}$ Lorentzian-Gaussian fit peaks. The $\mathrm{Cd} 3 \mathrm{~d}$ peak was deconvoluted using a Lorentzian-Gaussian function. The Lorentzian-Gaussian fit shows the existence of $\mathrm{Cd} 3 \mathrm{~d}$ (Lorentz fit 1) and $\mathrm{Cd} 3 \mathrm{~d}_{5 / 2}$ (Lorentz fit 2) bands which suggest that $\mathrm{Cd}$ is bound directly to Se at this two spin-orbital to form the CdSe QDs core. The Cd 3d band with a binding energy of $404.8 \mathrm{eV}$ [11] appears consistently in the $0-90 \mathrm{~min}$ XPS scan. The $\mathrm{Cd} 3 \mathrm{~d}_{5 / 2}$ band peak fitted to have a binding energy ranging from 405.3 to $406.1 \mathrm{eV}$, which is quite near to the value that was reported as $405.8 \mathrm{eV}( \pm 2)$ [19]. The position of Lorentz fit 1 shows that the $\mathrm{Cd} 3 \mathrm{~d}$ band appeared to have a consistent binding energy of $404.8 \mathrm{eV}$ for each scan, whereas $\mathrm{Cd} 3 \mathrm{~d}_{5 / 2}$ (Lorentz fit 2) appeared to have a slight chemical shift ranging between 405.3 to $406.1 \mathrm{eV}$. In addition, the $\mathrm{Cd} 3 \mathrm{~d}_{5 / 2}$ band was observed to appear in the Lorentz fit for the $0.5,1,5,16,46$, and 90 min sample scans and not in the $0 \mathrm{~min}$ and $1 \mathrm{~min}$ scans. This suggested that $0.5,1,5,16,46$, and $90 \mathrm{~min}$ Mn-doped CdSe QDs have an unpaired electron at the Cd $3 \mathrm{~d}$ band with a total electronic angular momentum (j) of 5/2 for the multiple spin-orbital splitting to occur. The values of $j$ are described in Equation (1).

$$
j=l+s
$$


where,

$l=$ total orbital angular/angular quantum number ( $>1$ for $\mathrm{p}, \mathrm{d}$ and $\mathrm{f}$ orbitals)

$s=$ spin momenta/spin quantum number $( \pm 1 / 2)$

Because $l$ is larger than zero for $\mathrm{d}$ orbital, the orbital line has the tendency to split into a doublet. For $3 d$ orbital, the doublet will be $3 d_{3 / 2}(s=-1 / 2)$ which indicates that the orbital is spinning down and $3 d_{5 / 2}(s=+1 / 2)$ indicates that the orbital is spinning up. Therefore, the spinning up orbital is dominant in the CdSe Mn-doped QDs orbital splitting system [11]. In contrast, there was no spin-orbital interaction in the 0 and 0.2 min samples, therefore the energy contained in the $\mathrm{Cd} 3 \mathrm{~d}$ spin-orbital are equal before and after the photoemission process. This is likely due to the increase in quantum yield on the surface of the atom as the size of the QDs decreases down to $5 \mathrm{~nm}$.

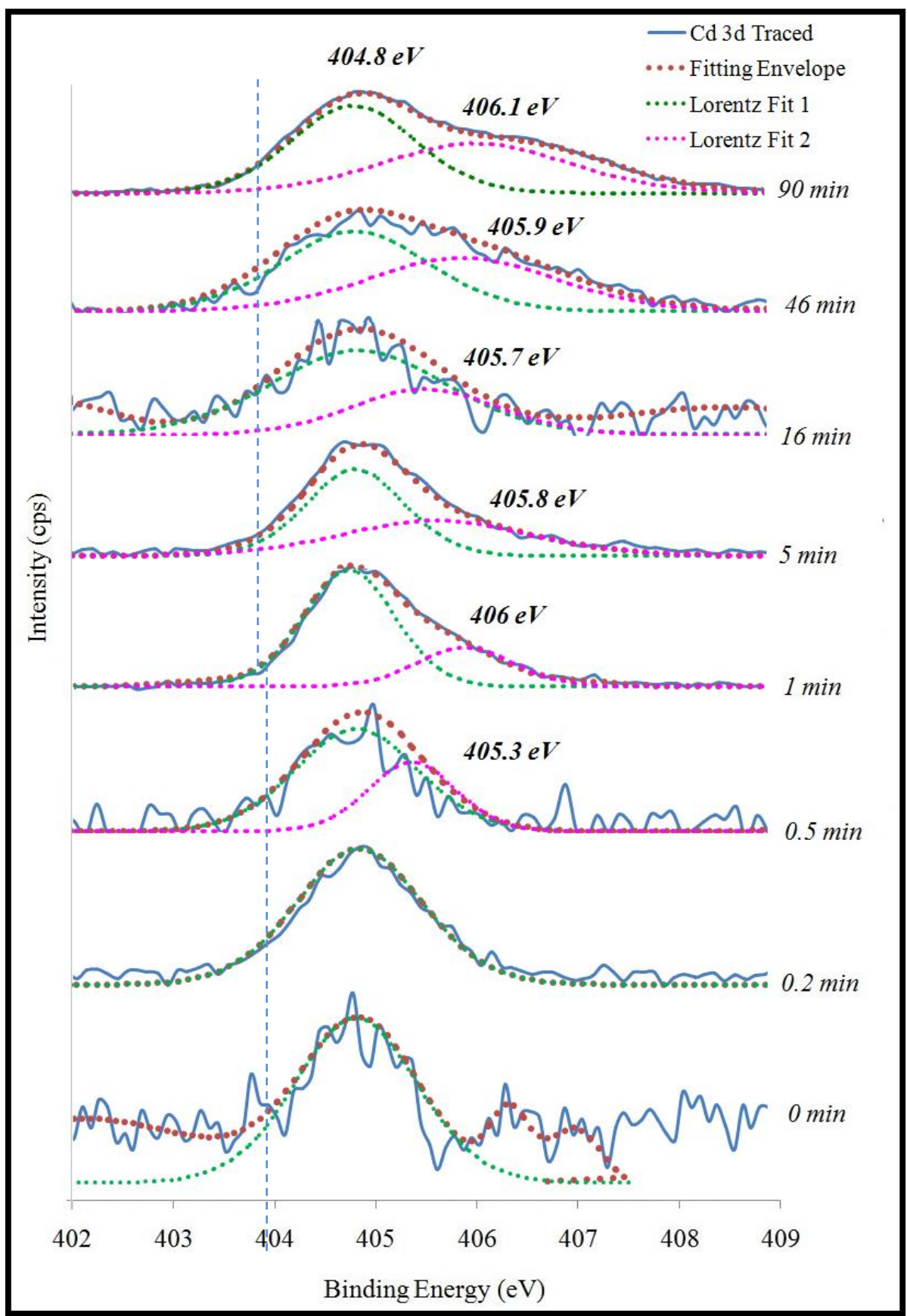

Figure 6. XPS scan of Cd 3d traced for Mn-doped CdSe QDs samples. 
Figure 7 shows the individual XPS peaks of Se 3d and its Lorentz fits. Contrary to the Cd 3d results, no obvious shift of original peak was observed. On the other hand, the Lorentz fit 1 shifted to a smaller binding energy $(404.2 \mathrm{eV})$ for the $0 \mathrm{~min}$ and $0.2 \mathrm{~min}$ samples, which also may be due to the function of QDs size in the chemical shift. Lorentz fit 2 shows readings ranging from 54.9 to $56.7 \mathrm{eV}$, excluding the $46 \mathrm{~min}$ and $16 \mathrm{~min}$ samples. XPS spectra for 0 min samples fitted to have extra Lorentz fit at $56.7 \mathrm{eV}$. Lorentz fit 2 and 3 indicated the influence of the Se $3 \mathrm{~d}_{5 / 2}$ band in the binding of CdSe QDs. As in Figure 5, the spinning up orbital dominates the Se system, excluding 46 min and 16 min, which may be due to the absence of spinning orbital interaction [14]. Interestingly, in the 0 min samples, the Se $3 \mathrm{~d}$ peak exhibits three Lorentz fitting features, $54.4 \mathrm{eV}$ corresponds to the Se $3 \mathrm{~d}$ band, and 55.2 and $56.7 \mathrm{eV}$ correspond to $3 \mathrm{~d}_{5 / 2}$, which is assumed to be due to the band structure complexation due to the higher density of lattice strain of core CdSe QDs where the Se atom is mainly located.

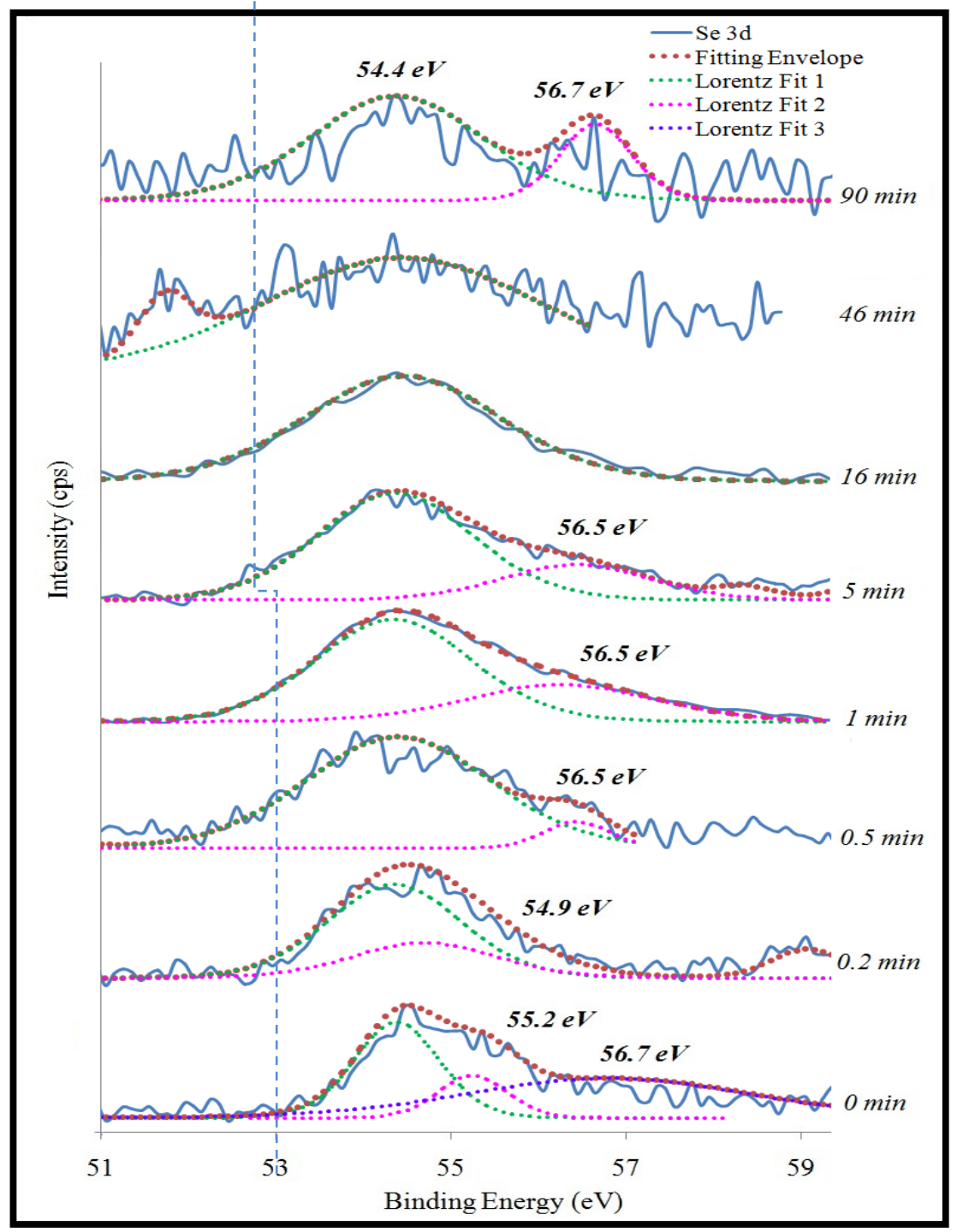

Figure 7. The XPS scan of Se traced for Mn-doped CdSe QDs at various reaction times.

Figure 8 shows the Mn 2p traced for Mn-doped CdSe QDs, which is crucial to prove the presence of Mn acts as a shell in core-shell Mn-doped CdSe QDs samples. The Lorentz fit 1, which corresponds to the Mn 2p band with a binding energy of $640.7 \mathrm{eV}$ is well fit in each sample. Lorentz Fit 2 with binding energy ranging from 642.2 to $645.9 \mathrm{eV}$ in each XPS scan initially created a shouldering peak and eventually shifted to create another defined peak as the QDs size increased. The role of the Mn 
shell as a lattice strain inducer may change with the growth in the size of the QDs. Moreover, the presence of Lorentz fit 3 and 4 for the 0 min sample suggests there are extreme changes in the lattice parameter at critically small QDs, which may be induced by the Mn shell. Overall, the intensity of the Mn 2p peaks declined as the size of the QDs increased. This could be due to the decrease in the thickness of the Mn shell as the size of the QDs increases, which is related to the function of Mn ion concentration with reaction time [16].

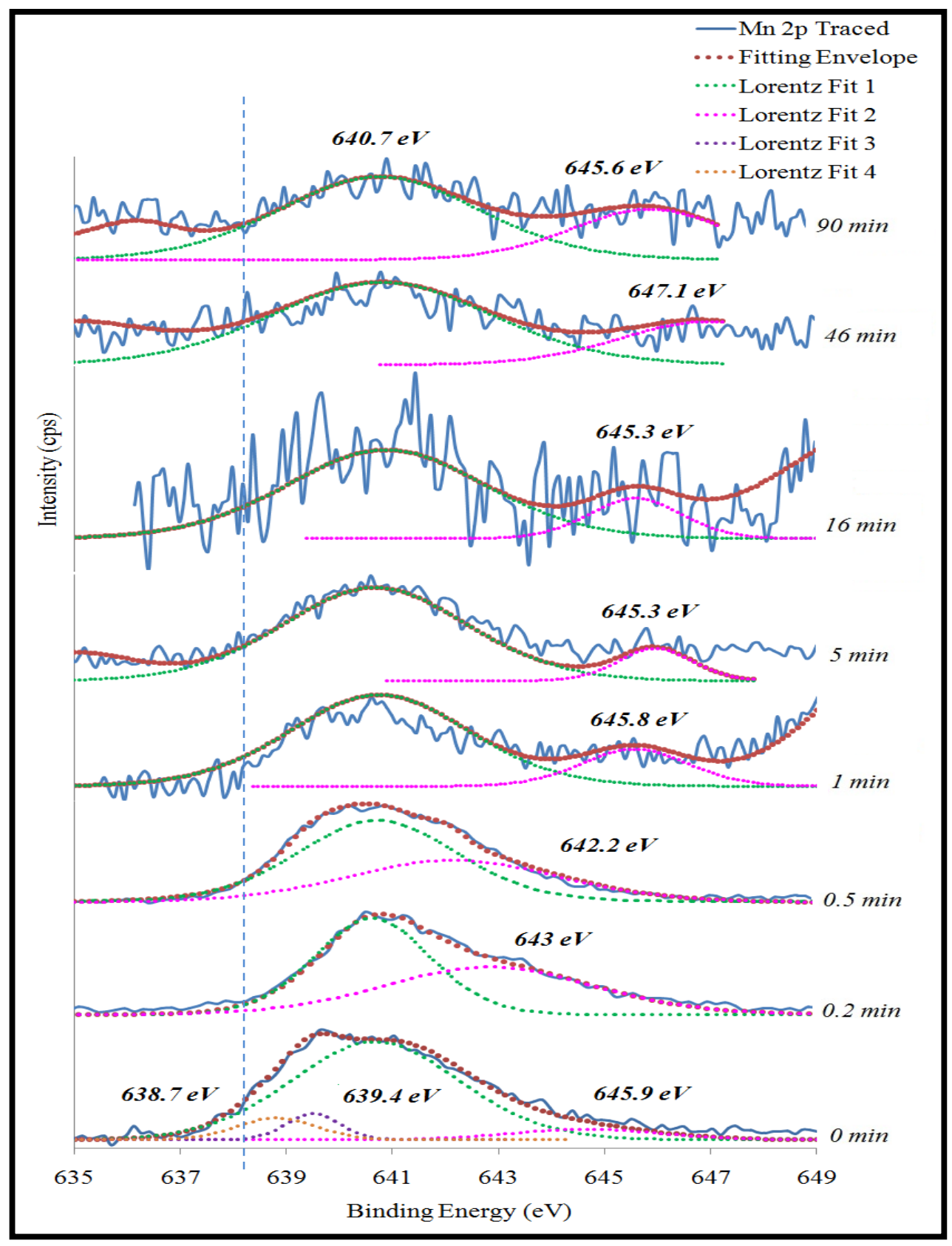

Figure 8. XPS scan for Mn traced for Mn-doped CdSe QDs at various reaction times.

The oxidation states of an exciting element are generally reported to be 2 for $\mathrm{Cd}, \pm 2,4$ and 6 for Se [16] and 7, 6, 4, 2 and 3 for Mn [16]. Figure 9 shows the relation between the Mn-doped CdSe QDs size with Cd 3d, Se 3d, and Mn 2p XPS peak intensity. Traced Cd 3d element shows the high values of peak intensity compared to Se $3 \mathrm{~d}$ and $\mathrm{Mn} 2 \mathrm{p}$. This is due to the high concentration of Cd atoms on the surface of QDs, which are presented as a Cd-oleate that acts as a ligand, and Cd atoms that 
are near the core-shell intermediate. These also suggest that $\mathrm{Cd}$ has an important role as the major electron donor in the Mn-doped CdSe QDs system. The relationship between the XPS peak intensity and the ion or atom concentration is strongly supported by Equation (2) where the electron intensity $(I)$ presented by XPS peak are directly proportional to the concentration of the atom or ion on the samples $(\rho)$, especially on the surface of the samples $[19,20]$.

$$
I=J \rho \sigma K \lambda
$$

where,

$$
\begin{aligned}
& I=\text { Electron intensity } \\
& J=\text { Photon flux } \\
& \rho=\text { Concentration of the atom or ion in the solid } \\
& \sigma=\text { Cross-section for photoelectron production } \\
& K=\text { Instrumental factors } \\
& \lambda=\text { Electron attenuation length }
\end{aligned}
$$

In addition, the intensity of $\mathrm{Cd} 3 \mathrm{~d}$ peaks was observed to decrease from 3364 to $2000 \mathrm{cps}$ as the QDs size increases. This may be due to the increases in electron concentration that leave the CdSe Mn-doped QDs solid samples after been irradiated by X-ray as the size of QDs became smaller. The smaller QDs have a larger surface area thus, larger sample area is exposed to the $\mathrm{x}$-ray, resulting in a higher concentration of electron emitted from the QDs surface [16]. A great drop in peak intensity was observed for the 0 to $46 \mathrm{~min}$ samples and became stable in the final stages (46 min and $90 \mathrm{~min}$ ). The influence of QDs size on the peak intensity was less pronounced as the size became larger than $12 \mathrm{~nm}$.

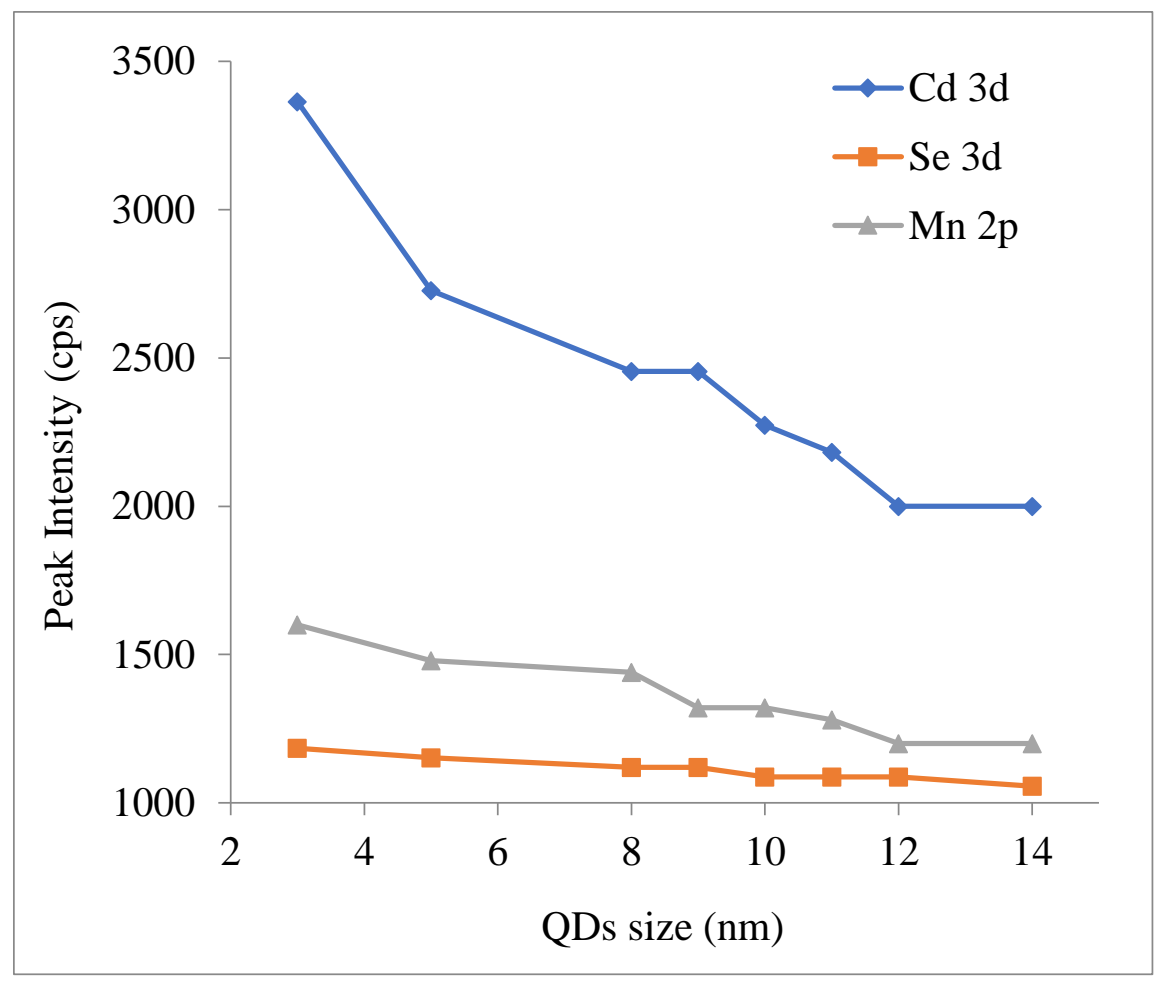

Figure 9. Relation between peak intensity and the variation in size of Mn-doped CdSe QDs.

Another interesting feature of XPS spectra is the peak broadening. Table 1 tabulated the full width at half maximum (FWHM) of Cd 3d, Se 3d, and Mn 2p peaks. The FWHM of Cd 3d ranged between 
1.3 and $1.5 \mathrm{eV}$ for 0 to $5 \mathrm{~min}$ reaction time samples, but then the FWHM values show a clear increase in the $16 \mathrm{~min}$ to 90 min samples. Se 3d and Mn 2p show narrowly distributed FWHM values for 0 min to $16 \mathrm{~min}$ samples. The FWHM values increased for the $46 \mathrm{~min}$ and $90 \mathrm{~min}$ samples. This increment may be relative to the QDs size increment, especially for $\mathrm{Cd} 3 \mathrm{~d}$ since $\mathrm{Cd}$ is predicted to be the larger carrier donor at the surface of QDs. Besides, increases in the energy band gap were inversely proportional to the QDs. To elaborate further, Equation (2) shows that the relationship between the energy band gap $(E)$ and the carrier effective mass $\left(m^{*}\right)$ are also inversely proportional, where $\hbar$ and $k$ are constants factors.

$$
E=\frac{\hbar^{2} k^{2}}{2 m^{*}}
$$

The charge carrier lifetime is highly dependent on the charge carrier concentration since it is also proportional to the effective mass of the carrier. Peak broadening with a decrement in the energy band gap may be due to the increase in carrier lifetime. According to the Heisenberg's uncertainty principle [16], the core-hole, which is created by the incident photon has a finite lifetime, will create a broadening effect in the XPS peak. This broadening is well known as natural or lifetime broadening which is also the minimum attainable peak width. The collision of $\mathrm{Cd}$, Se and $\mathrm{Mn}$ atoms with each other, whether between the same or different elements, will create distorted energy levels and results in a shorter lifetime of the excited state. This collision effect leads to the broadening of the XPS peak (collision broadening). Both of these broadening effects match a Lorentzian profile [16].

Table 1. Full width at half maximum of the XPS peak of Mn-doped CdSe QDs for different reaction times.

\begin{tabular}{cccc}
\hline Reaction Time & \multicolumn{3}{c}{ FWHM (eV) } \\
\cline { 2 - 4 } (min) & Cd 3d & Se 3d & Mn 2p \\
\hline 0 & 1.5 & 2.2 & 2.3 \\
0.2 & 1.4 & 2.2 & 2.2 \\
0.5 & 1.3 & 2.6 & 2.3 \\
1 & 1.3 & 2.7 & 2.3 \\
5 & 1.3 & 2.6 & 2.4 \\
16 & 2.2 & 2.7 & 2.5 \\
46 & 2.5 & 3.6 & 3.0 \\
90 & 2.3 & 4.1 & 5.1 \\
\hline
\end{tabular}

A broad QD size distribution may also lead to the XPS peak broadening. The variation in the defect density due to the QD size distribution leads to the variations in band bending. This band bending will produce broadened peaks. This discussion is supported by the QDs size distribution histogram, which shows the broad size distribution QDs in the $46 \mathrm{~min}$ and $90 \mathrm{~min}$ samples compared to the others [16].

Rani et al. reported that increases in dopant concentration results in peaks shifting towards higher energy values, which suggests that the dopant concentration contributes to the lattice modification in the CdSe QDs compound [10]. However, the effect of incorporated dopant on QDs is limited at a certain dopant concentration. The II-VI QDs electronic properties were reported to deteriorate after a specific dopant concentration was reached [10]. It is important that the dopant concentration and solubility limit in each compound of QDs be studied further.

\section{Conclusions}

Mn-doped CdSe QDs were synthesized with a narrow QDs size distribution using the inverse Micelle method and with quasi-sphere shaped QDs. Mn-doped CdSe QDs have a high-quality zinc blende crystal structure with the tetrahedral plane (001) doped with Mn ion. Mn-doping is predicted to optimize the surface passivation of anionic and cationic surface trap states that organic ligands cannot overcome. Furthermore, $\mathrm{Mn}^{2+}$ incorporation adds strain to the CdSe QDs lattice, which allows 
the tuning of inter-band energy structure. This ensures the tunability of its optical and electronic properties. The surface composition of the Mn-doped CdSe QDs is shown by the incorporation of Mn into CdSe lattice. The addition of strain to the lattice of the core CdSe QDs is prominent at the interface of QDs, which is highly incorporated by Mn dopants.

Author Contributions: N.A.H.-Date curstion, format analisis, investugation, project administration, writing - original draft; M.R.J.—Format analisis, resources, supervision; Y.A.W.—Date curation; Z.Z.C.—Date curation; O.A.—Date curation; S.S.—Writing—review \& editing; I.A.B.-Funding acquisition; T.M.Y.K.—Funding acquisition; S.K.-Funding acquisition.

Funding: This research was funded by King Khalid University grant number R.G.P. 2/11/39 and University of Malaya grant number RU001-2018, RU001-2019 and ST018-2018. The APC was funded by University of Malaya.

Acknowledgments: The authors extend their appreciation to the Deanship of Scientific Research at King Khalid University for funding this work through research groups program under grant number (R.G.P. 2/11/39). This work was also financially supported by University of Malaya Research Grant (RU001-2018, RU001-2019 and ST018-2018).

Conflicts of Interest: The authors declare no conflict of interest.

\section{References}

1. Talapin, D.V.; Rogach, A.L.; Kornowski, A.; Haase, M.; Weller, H. Highly Luminescent Monodisperse CdSe and CdSe/ZnS Nanocrystals Synthesized in a Hexadecylamine-Trioctylphosphine Oxide-Trioctylphospine Mixture. Nano Lett. 2001, 1, 207-211. [CrossRef]

2. Devi, L.S.; Devi, K.N.; Sharma, B.I.; Sarma, H.N. Influence of Mn doping on structural and optical properties of CdS nanoparticles. Indian J. Phys. 2014, 88, 477-482. [CrossRef]

3. Kwak, W.-C.; Kim, T.G.; Chae, W.-S.; Sung, Y.-M. Tuning the energy bandgap of CdSe nanocrystals via Mg doping. Nanotechnology 2007, 18, 205702. [CrossRef]

4. Sung, Y.-M.; Kwak, W.-C.; Kim, T.G. Coarsening Kinetics of Mn-Doped CdSe Nanocrystals. Cryst. Growth Des. 2008, 8, 1186-1190. [CrossRef]

5. Kwak, W.-C.; Sung, Y.-M.; Kim, T.G.; Chae, W.-S. Synthesis of Mn-doped zinc blende CdSe nanocrystals. Appl. Phys. Lett. 2007, 90, 173111. [CrossRef]

6. Perdew, J.P.; Burke, K.; Ernzerhof, M. Generalized gradient approximation made simple. Phys. Rev. Lett. 1996, 77, 3865-3868. [CrossRef] [PubMed]

7. Erwin, S.C.; Zu, L.; Haftel, M.I.; Efros, A.L.; Kennedy, T.A.; Norris, D.J. Doping semiconductor nanocrystals. Nature 2005, 436, 91-94. [CrossRef] [PubMed]

8. Alivisatos, A.P. Semiconductor clusters, nanocrystals, and quantum dots. Science 1996, 271, $933-937$. [CrossRef]

9. Mikulec, F.V. Organometallic synthesis and spectroscopic characterization of manganese-doped CdSe nanocrystals. J. Am. Chem. Soc. 2000, 122, 2532-2540. [CrossRef]

10. Hamizi, N.A.; Johan, M.R. Synthesis and size dependent optical studies in CdSe quantum dots via inverse micelle technique. Mater. Chem. Phys. 2010, 124, 395-398. [CrossRef]

11. Valais, I.; Michail, C.; Fountzoula, C.; Tseles, D.; Yannakopoulos, P.; Nikolopoulos, D.; Bakas, A.; Fountos, G.; Saatsakis, G.; Sianoudis, I.; et al. On the response of alloyed ZnCdSeS quantum dot films. Results Phys. 2017, 7, 1734-1736. [CrossRef]

12. Jiang, F.; Muscat, A.J. Ligand-controlled growth of ZnSe quantum dots in water during Ostwald ripening. Langmuir 2012, 28, 12931-12940. [CrossRef] [PubMed]

13. Dongzhi, Y.; Qifan, C.; Shukun, X. Synthesis of CdSe/CdS with a simple non-TOP-based route. J. Lumin. 2007, 126, 853-858.

14. Agostinelli, E.; Battistoni, C.; Fiorani, D.; Mattogno, G.; Nogues, M. An XPS study of the electronic structure of the $\mathrm{Zn}_{\mathrm{x}} \mathrm{Cd}_{1-\mathrm{x}} \mathrm{Cr}_{2}(\mathrm{X}=\mathrm{S}$, Se) spinel system. J. Phys. Chem. Solids 1989, 50, 269-272. [CrossRef]

15. Ivanov-Ehmin, B.; Nevskaya, N.; Zajtsev, B.; Ivanova, T. Synthesis and properties of calcium and strontium hydroxomanganates (3). Zhurnal Neorg. Khimii 1982, 27, 3101-3104.

16. Dai, Q.; Sabio, E.M.; Wang, W.; Tang, J. Pulsed laser deposition of Mn doped CdSe quantum dots for improved solar cell performance. Appl. Phys. Lett. 2014, 104, 183901. [CrossRef]

17. Smith, A.M.; Nie, S. Semiconductor Nanocrystals: Structure, Properties, and Band Gap Engineering. Acc. Chem. Res. 2010, 43, 190-200. [CrossRef] [PubMed] 
18. Pokrant, S.; Whaley, K.B. Tight-Binding studies of surface effects on electronic structure of CdSe nanocrystals: the role of organic ligands, surface reconstruction, and inorganic capping shells. Eur. Phys. J. D 1999, 6, 255-267. [CrossRef]

19. Katari, J.B.; Colvin, V.L.; Alivisatos, A.P. X-ray photoelectron spectroscopy of CdSe nanocrystals with applications to studies of the nanocrystal surface. J. Phys. Chem. 1994, 98, 4109-4117. [CrossRef]

20. Gaarenstroom, S.; Winograd, N. Initial and final state effects in the ESCA spectra of cadmium and silver oxides. J. Chem. Phys. 1977, 67, 3500-3506. [CrossRef]

(C) 2019 by the authors. Licensee MDPI, Basel, Switzerland. This article is an open access article distributed under the terms and conditions of the Creative Commons Attribution (CC BY) license (http://creativecommons.org/licenses/by/4.0/). 